\title{
Synthesis of some novel 4-arylidene pyrazoles as potential antimicrobial agents
}

\author{
Poonam Khloya ${ }^{1}$, Pawan Kumar ${ }^{1}$, Arpana Mittal ${ }^{2}$, Neeraj K Aggarwal ${ }^{2}$ and Pawan K Sharma ${ }^{\text {* }}$
}

\begin{abstract}
Background: Pyrazole and pyrazolone motifs are well known for their wide range of biological activities such as antimicrobial, anti-inflammatory, and antitumor activities. The incorporation of more than one pharmacophore in a single scaffold is a well known approach for the development of more potent drugs. In the present investigation, a series of differently substituted 4-arylidene pyrazole derivatives bearing pyrazole and pyrazolone pharmacophores in a single scaffold was synthesized.

Results: The synthesis of novel 4-arylidene pyrazole compounds is achieved through Knovenagel condensation between 1,3-diaryl-4-formylpyrazoles and 3-methyl-1-phenyl-1H-pyrazol-5-(4H)-ones in good yields. All compounds were evaluated for their in vitro antimicrobial activity.

Conclusions: A series of 4-arylidene pyrazole derivatives was evaluated for their in vitro antimicrobial activity against two Gram-positive (Bacillus subtilis and Staphylococcus aureus) and two Gram-negative bacteria (Pseudomonas fluorescens and Escherichia coli), as well as two pathogenic fungal strains (Candida albicans and Saccharomyces cerevisiae). The majority of the compounds displayed excellent antimicrobial profile against the Gram-positive (B. subtilis and S. aureus), and some of them are even more potent than the reference drug ciprofloxacin.
\end{abstract}

Keywords: Pyrazole; Pyrazolone; Antibacterial activity; Antifungal activity; Sulfonamide

\section{Background}

Over the years, excessive use of antimicrobial drugs has led to a worldwide phenomenon of antibacterial resistance. This has resulted into an increase in morbidity and mortality, and has become a worldwide health issue. As a consequence, the development of new antimicrobial agents is in constant demand. The compounds bearing pyrazole nucleus are well known to exhibit versatile range of biological activities such as antimicrobial [1-4], antiinflammatory [5-7], antidepressant [8], antiviral [9], and antitumor activities [10]. Among these, 4-functionalized pyrazoles occupy a unique position in medicinal chemistry because of their association with antimicrobial [11], antiinflammatory [12], antiparasitic [13], and antitumor activities [14]. Pyrazol-5-(4H)-one also constitutes the core scaffold of various biologically active synthetic heterocyclic compounds which have been associated with some interesting pharmaceutical properties, including analgesic [15], antimicrobial [16,17], anti-inflammatory [18], antitumor

\footnotetext{
* Correspondence: pksharma@kuk.ac.in

'Department of Chemistry, Kurukshetra University, Kurukshetra 136119, India Full list of author information is available at the end of the article
}

[19], and cytotoxicity [20] properties. Understanding that the incorporation of both pyrazole and pyrazolone together in the same scaffold could provide novel compounds with interesting biological activities coupled with our continuing research interest in the field of 4functionalized pyrazole derivatives [12,21-23] and other biologically active synthetic heterocyclic compounds [24-27], we set out to undertake the synthesis of some novel 4-arylidene pyrazole derivatives bearing benzenesulfonamide moiety at the N1-position of the pyrazole ring (Scheme 1) as potential antimicrobial agents.

\section{Methods}

In vitro antibacterial activity

The agar well diffusion method [28] was used for the determination of antimicrobial activity of all the synthesized compounds. Overnight broth culture of the respective bacterial strains was adjusted to approximately $10^{8}$ colony forming units $(\mathrm{CFU} / \mathrm{mL})$ with sterile distilled water, and $100 \mu \mathrm{L}$ of diluted inoculum was spread over the petriplates containing $25 \mathrm{~mL}$ of nutrient agar media. Eight wells ( $8 \mathrm{~mm}$ in diameter) were made 


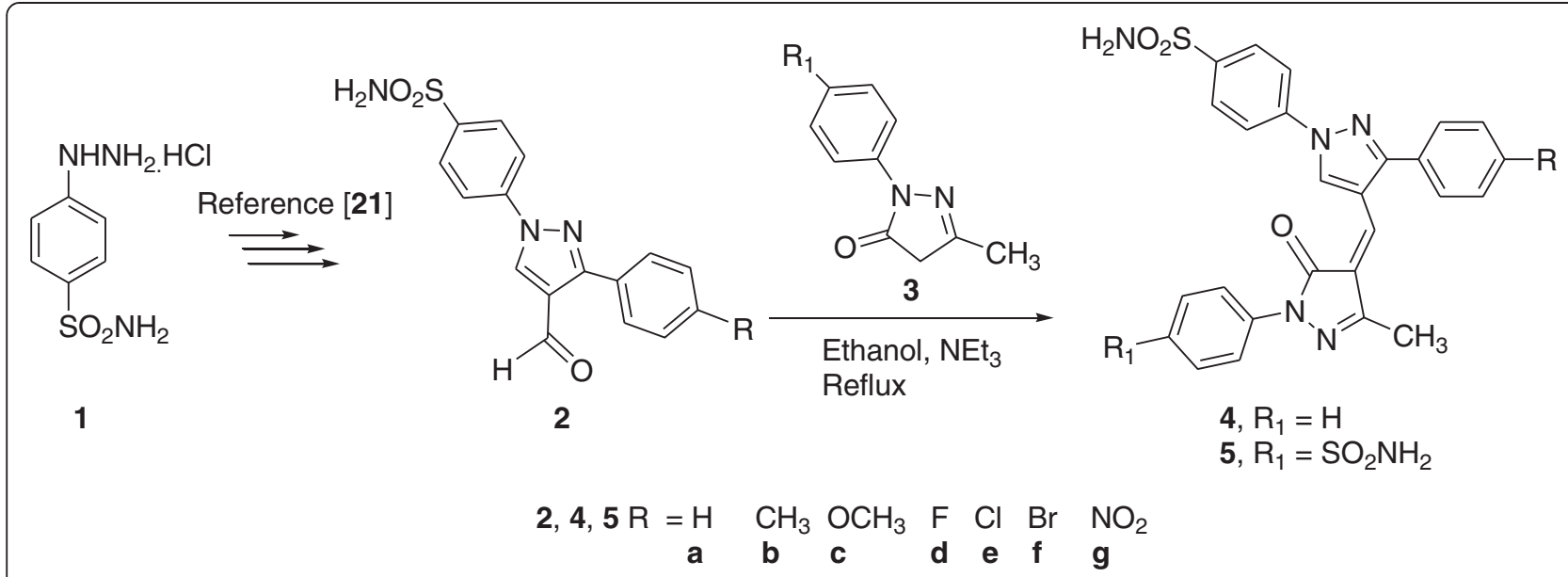

Scheme 1 Synthesis of 4-arylidene pyrazole derivatives 4 and 5 .

equidistant with each of the plates using a sterile cork borer. The test compounds were dissolved in dimethylsulfoxide (DMSO) and then the antimicrobial effect of the synthesized compounds was evaluated. The wells were filled with $100 \mu \mathrm{L}$ of the test compound having a concentration of $4.0 \mathrm{mg} / \mathrm{mL}$. The plates were incubated at $37^{\circ} \mathrm{C}$ for $48 \mathrm{~h}$. The antimicrobial activity was evaluated by measuring the zone of growth inhibition of bacteria surrounding the wells after 24 and $48 \mathrm{~h}$. Ciprofloxacin (4.0 $\mathrm{mg} / \mathrm{mL}$ ) served as the antibacterial control. DMSO was taken as the negative control which did not produce any significant zone of inhibition.

\section{Determination of minimum inhibitory concentration}

The minimum inhibitory concentration (MIC) against the tested bacteria was determined using the macrodilution tube method [29] as recommended by NCCLS (2000). The MIC is the lowest concentration of an antimicrobial compound, which will inhibit the visible growth of a microorganism after an overnight incubation. The MIC of each compound giving an inhibitory zone at a concentration of $4 \mathrm{mg} / \mathrm{mL}$ was also tested with the agar well diffusion method. Different concentrations (4,000 to $0.004 \mu \mathrm{g} /$ $\mathrm{mL}$ ) of a single compound were applied to the number of wells in the agar plates. The determinations were performed in triplicates, and the results were averaged.

\section{In vitro antifungal activity}

The agar well diffusion method was used for the determination of antimicrobial activity of the compounds. Overnight broth culture of the respective fungal strains was adjusted to approximately $10^{8} \mathrm{CFU} / \mathrm{mL}$ with sterile distilled water, and $100 \mu \mathrm{L}$ of diluted inoculum was spread over the petriplates containing $25 \mathrm{~mL}$ of Sabouraud's dextrose agar media ( $\mathrm{pH}$ 5.6). Eight wells ( $8 \mathrm{~mm}$ in diameter) were made equidistant with each of the plates using a sterile cork borer. The test compounds were dissolved in
DMSO and then the antimicrobial effect of the test compounds was tested. The wells were filled with $100 \mu \mathrm{L}$ of the test compound having a concentration $4 \mathrm{mg} / \mathrm{mL}$. The plates were incubated at $30^{\circ} \mathrm{C}$ for 48 to $72 \mathrm{~h}$. The antimicrobial activity was evaluated by measuring the zone of growth inhibition of fungi surrounding the wells after 48 and $72 \mathrm{~h}$. Fluconazole $(4 \mathrm{mg} / \mathrm{mL})$ served as the antifungal control. DMSO was taken as the negative control which did not produce any significant zone of inhibition. The experiments were performed in triplicates. The diameter of the fungal colonies was measured.

\section{Results and discussion Chemistry}

The synthetic route used to synthesize the target 4arylidene pyrazole derivatives ( $\mathbf{4}$ and $\mathbf{5}$ ) is outlined in Scheme 1. 4-Formyl pyrazoles (2) were synthesized using our earlier reported procedure [21], while 3-methyl-1aryl-1H-pyrazol-5-(4H)-ones (3) was prepared by condensation of ethylacetoacetate with appropriate hydrazine [30,31]. Finally, base-catalyzed Knoevenagel condensation of appropriately substituted pyrazol-5-(4H)-ones (3) with various substituted 4-formylpyrazoles (2) in ethanol containing catalytic amount of triethylamine afforded the target 4-arylidene pyrazole derivatives (4 and 5$)$ in good yield. Spectral data (IR, ${ }^{1} \mathrm{H}$ NMR, and mass) of the newly synthesized compounds $\mathbf{4 a - g}$ and $\mathbf{5 a - g}$ were in full agreement with the proposed structures. In the ${ }^{1} \mathrm{H}$ NMR spectra of $\mathbf{4}$ and $\mathbf{5}$, the $\mathrm{C}=\mathrm{CH}$ proton displayed more downfield signal in the range $\delta 10.18$ to 10.25 . Besides this, $\mathrm{C}_{5}-\mathrm{H}$ of the pyrazole ring resonates at around $\delta 7.51$ to 7.63. The IR spectra of $\mathbf{4}$ and $\mathbf{5}$ showed a characteristic absorption band around 1,674 to $1,682 \mathrm{~cm}^{-1}$ that was assigned to the $\mathrm{C}=\mathrm{O}$ stretching, while the two absorptions bands around 1,304 to 1,335 and 1,149 to $1,165 \mathrm{~cm}^{-1}$ which further supported the proposed structures of newly synthesized compounds displayed the $\mathrm{SO}_{2}$ stretchings. 


\section{Biological evaluation}

\section{In vitro antibacterial activity}

All the synthesized compounds (4 and 5 ) were screened for their in vitro antibacterial activity against the four pathogenic bacteria, Bacillus subtilis (microbial-type culture collection (MTCC) 121) and Staphylococcus aureus (MTCC 96) representing the Gram-positive bacteria, and Pseudomonas fluorescens (MTCC 1749) and Escherichia coli (MTCC 1652) representing the Gram-negative bacteria (Table 1), by agar well diffusion method [28] using ciprofloxacin as the reference drug. The MIC measurements were performed using a macrodilution method [29] (Table 1).

The results revealed that all the tested compounds showed variable antibacterial activity against the Grampositive as well as the Gram-negative bacteria. Among the tested compounds, the antibacterial activity of compounds $4 \mathbf{a}$ and $5 \mathbf{a}$ with a zone of inhibition of $28 \mathrm{~mm}$ (MIC $0.04 \mu \mathrm{g} / \mathrm{mL}$ ) was found to be better than that of the reference drug ciprofloxacin with a zone of inhibition of $26 \mathrm{~mm}$ (MIC $0.4 \mu \mathrm{g} / \mathrm{mL}$ ) against B. subtilis. Compounds $\mathbf{4 f}$ and $\mathbf{5 d}$ also displayed appreciable activity with a zone of inhibition of $24 \mathrm{~mm}$ (MIC $0.4 \mu \mathrm{g} / \mathrm{mL}$ ) and $22 \mathrm{~mm}$ (MIC $4.0 \mu \mathrm{g} / \mathrm{mL}$ ), respectively, against $B$. subtilis. Compound 5a with two sulfonamide groups was found to be the most effective against $S$. aureus, showing a maximum zone of inhibition of $30 \mathrm{~mm}$ (MIC $0.04 \mu \mathrm{g}$ / mL). 4a also showed antibacterial activity with a zone of inhibition of $26 \mathrm{~mm}$ (MIC $0.4 \mu \mathrm{g} / \mathrm{mL}$ ) comparable to the reference drug ciprofloxacin with a zone of inhibition of $26 \mathrm{~mm}$ (MIC $0.4 \mu \mathrm{g} / \mathrm{mL}$ ) against S. aureus. Six more compounds (4b-c, $\mathbf{4 f}$, and $\mathbf{5 b}$-d) were found to possess appreciable antibacterial activity with a zone of inhibition greater than $20 \mathrm{~mm}$ against S. aureus. Interestingly, compounds $\mathbf{4 a}$ and $\mathbf{5 a}$, both with an unsubstituted phenyl ring at $\mathrm{C}-3$ of pyrazole, displayed a tenfold MIC $(0.04 \mu \mathrm{g} / \mathrm{mL})$ better than the standard drug ciprofloxacin against $B$. subtilis. A comparison within each series suggested that any substituent on the phenyl ring placed at the 3-position of the pyrazole moiety has a negative effect on the antibacterial activity against Gram-positive bacteria, as best results were seen with the naked phenyl ring in each series (compare $\mathbf{4 a}$ with $\mathbf{4 a - g}$, and $\mathbf{5 a}$ with 5a-g; Table 1). No definite trend was discernable that could lead to draw a correlation of the activities between series $\mathbf{4}$ and $\mathbf{5}$.

Against Gram-negative bacteria (P. fluorescens), only compound 5e showed a significant activity with a zone of inhibition of $25 \mathrm{~mm}$ (MIC $0.4 \mu \mathrm{g} / \mathrm{mL}$ ) comparable to the standard drug ciprofloxacin (zone of inhibition 23 $\mathrm{mm})$, albeit with a tenfold better MIC. Against E. coli, five compounds (4e, $4 \mathbf{g} \mathbf{5 a}, \mathbf{5 d}$, and $5 \mathbf{g}$ ) showed good antibacterial activity with a zone of inhibition of $20 \mathrm{~mm}$. However, none of the compounds were found to be as effective as the standard drug ciprofloxacin against $E$. coli (Table 1). Thus, it can be concluded that the synthesized compounds are more effective against the Grampositive bacteria than the Gram-negative bacteria.

\section{In vitro antifungal activity}

All the synthesized compounds were also evaluated for their in vitro antifungal activity against the two pathogenic

Table 1 In vitro antibacterial activity and MIC of compounds 4 and 5 using agar well diffusion method

\begin{tabular}{|c|c|c|c|c|}
\hline \multirow[t]{2}{*}{ Compound $^{a}$} & \multicolumn{4}{|c|}{ Diameter of zone of inhibition in $\mathrm{mm}(\mathrm{MIC})^{b}$} \\
\hline & B. subtilis & S. aureus & P. fluorescens & E. coli \\
\hline $4 a$ & $28 \pm 0.00(0.04)$ & $26 \pm 0.30(0.4)$ & $14 \pm 0.08(400)$ & $14 \pm 0.46(400)$ \\
\hline $4 b$ & $20 \pm 0.19(4.0)$ & $24 \pm 0.50(0.4)$ & $15 \pm 0.09(400)$ & $14 \pm 0.23(400)$ \\
\hline $4 c$ & $16 \pm 0.19(40)$ & $24 \pm 0.32(0.4)$ & - & $14 \pm 0.23(400)$ \\
\hline $4 d$ & $16 \pm 0.00(40)$ & $12 \pm 0.19(400)$ & - & $14 \pm 0.43(400)$ \\
\hline $4 \mathrm{e}$ & $12 \pm 0.40(400)$ & $16 \pm 0.22(40)$ & - & $20 \pm 0.30(4)$ \\
\hline $4 f$ & $24 \pm 0.07(0.4)$ & $20 \pm 0.37(4.0)$ & $14 \pm 0.14(400)$ & $18 \pm 0.33(40)$ \\
\hline $4 g$ & $14 \pm 0.23(400)$ & $12 \pm 0.10(400)$ & - & $20 \pm 0.34(4)$ \\
\hline $5 a$ & $28 \pm 0.09(0.04)$ & $30 \pm 0.15(0.04)$ & - & $20 \pm 0.32(4)$ \\
\hline $5 b$ & $16 \pm 0.32(40)$ & $25 \pm 0.42(0.4)$ & $16 \pm 0.12(40)$ & $14 \pm 0.50(400)$ \\
\hline $5 c$ & $14 \pm 0.18(400)$ & $22 \pm 0.24(40)$ & & $18 \pm 0.54(40)$ \\
\hline $5 d$ & $22 \pm 0.09(4.0)$ & $22 \pm 0.20(4.0)$ & $16 \pm 0.23(40)$ & $20 \pm 0.12(4)$ \\
\hline $5 e$ & $14 \pm 0.00(400)$ & $16 \pm 0.00(40)$ & $25 \pm 0.13(0.4)$ & $18 \pm 0.23(40)$ \\
\hline $5 f$ & $12 \pm 0.33(400)$ & $16 \pm 0.36(40)$ & - & $16 \pm 0.43(40)$ \\
\hline $5 g$ & $14 \pm 0.32(400)$ & $12 \pm 0.30(400)$ & $14 \pm 0.43(400)$ & $20 \pm 0.21$ \\
\hline Ciprofloxacin & $26 \pm 0.025(0.4)$ & $26 \pm 0.45(0.4)$ & $23 \pm 0.42(4.0)$ & $25 \pm 0.44(0.4)$ \\
\hline
\end{tabular}


fungal strains Candida albicans (MTCC 227) and Saccharomyces cerevisiae (MTCC 170) by agar well diffusion method (Table 2). Fluconazole was used as the reference drug. Most of the tested compounds in each series ( $\mathbf{4}$ and 5) showed moderate to good antifungal activity. Compound $\mathbf{4 g}$ was found to be as effective as the standard drug, with a zone of inhibition of $16 \mathrm{~mm}$ against $C$. albicans. Against S. cerevisiae, $\mathbf{4 a}$ and $\mathbf{5 b}$ (zone of inhibition $28 \mathrm{~mm}$ ) were found to be the most effective and were better than the standard drug fluconazole (zone of inhibition $24 \mathrm{~mm}$ ), while the three other compounds $(\mathbf{4 d}, \mathbf{5 e}$, and $\mathbf{5 g}$ ) were found to possess good antifungal activity. Interestingly, some of the newly synthesized compounds $(4 \mathbf{a}, 5 \mathbf{b}, 5 \mathbf{e}$, and $5 \mathrm{~g}$ ) showed multifold reduction in the MIC values against $S$. cerevisiae, making the new derivatives attractive agents for further evaluation.

\section{Experimental}

The melting points were determined in open capillaries in an electrical apparatus and were uncorrected. The IR spectra in $\mathrm{KBr}$ were recorded with the $\mathrm{ABB}$ MB3000 DTGS IR instrument. The ${ }^{1} \mathrm{H}$ NMR spectra were recorded on Bruker instrument (Bruker Scientific Instruments, MA, USA) at $300 \mathrm{MHz}$, taking DMSO- $d_{6}$ as the solvent. The chemical shifts are expressed in $\delta$, ppm. The mass spectra (DART-MS) were recorded on a JEOL AccuTOF JMS-T100LC mass spectrometer having a direct analysis in real time (DART) source in the $\mathrm{ES}^{+}$mode. The purity of the compounds was checked using ${ }^{1} \mathrm{H}$

Table 2 In vitro antifungal activity and MIC of compounds 4 and 5 using agar well diffusion method

\begin{tabular}{ccc}
\hline Compound $^{\mathbf{a}}$ & \multicolumn{2}{c}{ Diameter of zone of inhibition in $\mathbf{~ m m ~ ( M I C )}$} \\
\cline { 2 - 3 } & Candida albicans & Saccharomyces cerevisiae \\
\hline $\mathbf{4 a}$ & $12 \pm 0.43(400)$ & $28 \pm 0.15(0.04)$ \\
$\mathbf{4 b}$ & $12 \pm 0.56(400)$ & $16 \pm 0.38(40)$ \\
$\mathbf{4 c}$ & $12 \pm 0.54(400)$ & $16 \pm 0.40(40)$ \\
$\mathbf{4 d}$ & $14 \pm 0.14(400)$ & $20 \pm 0.45(4.0)$ \\
$\mathbf{4 e}$ & $12 \pm 0.45(400)$ & $18 \pm 0.16(40)$ \\
$\mathbf{4 f}$ & $12 \pm 0.32(400)$ & $16 \pm 0.27(40)$ \\
$\mathbf{4 g}$ & $16 \pm 0.27(40)$ & $18 \pm 0.48(40)$ \\
$\mathbf{5 a}$ & $12 \pm 0.32(400)$ & $16 \pm 0.00(40)$ \\
$\mathbf{5 b}$ & $12 \pm 0.33(400)$ & $28 \pm 0.28(0.04)$ \\
$\mathbf{5 c}$ & - & $16 \pm 0.00(40)$ \\
$\mathbf{5 d}$ & $12 \pm 0.23(400)$ & $18 \pm 0.18(40)$ \\
$\mathbf{5 e}$ & $13 \pm 0.37(400)$ & $22 \pm 0.44(4.0)$ \\
$\mathbf{5 f}$ & - & $16 \pm 0.30(40)$ \\
$\mathbf{5 g}$ & $12 \pm 0.43(400)$ & $20 \pm 0.10(4.0)$ \\
Fluconazole & $16 \pm 0.45(40)$ & $24 \pm 0.50(40)$ \\
\hline
\end{tabular}

MIC $(\mu \mathrm{g} / \mathrm{mL})$, minimum inhibitory concentration. Hyphen denotes no activity. ${ }^{\text {a }}$ The concentration is $4.0 \mathrm{mg} / \mathrm{mL}$. ${ }^{\text {b }}$ The values, including the diameter of the well $(8 \mathrm{~mm})$, are means of the three replicates.
NMR and thin layer chromatography on silica gel plates, using a mixture of petroleum ether and ethyl acetate as the eluent. Iodine or UV lamp was used as a visualizing agent. In the following section, these abbreviations are used: ' $\mathrm{s}$ ' for singlet, ' $\mathrm{m}$ ' for multiplet, and 'ex' for exchangeable proton are used for the NMR assignments; ' $\mathrm{s}$ ' for strong and ' $\mathrm{m}$ ' for medium are used for the IR assignments.

\section{General procedure for the conversion of 4-formylyrazole} into 4-arylidene pyrazole derivatives ( 4 and 5 )

To a solution of 4-formylpyrazoles, compound $2(1 \mathrm{mmol})$ in ethanol was added to the appropriate pyrazolone 3 ( $1 \mathrm{mmol}$ ) followed by a catalytic amount of triethylamine and refluxed the resulting reaction mixture for 6 to $7 \mathrm{~h}$. After the completion of the reaction, the solution was reduced to $1 / 4$ of its volume and cooled to room temperature. The solid separated out was filtered, washed with water $(100 \mathrm{~mL})$ followed by cold ethanol $(10 \mathrm{~mL})$, and crystallized from ethanol to afford the target compounds 4 and 5 .

\section{4-\{4-[(3-methyl-5-oxo-1-phenyl-1,5-dihydro-4H-pyrazol-4- ylidene)methyl]-3-phenyl-1H-pyrazol-1-yl\} benzenesulfonamide (4a)}

M.p. $272^{\circ} \mathrm{C}$ to $275^{\circ} \mathrm{C}$, yield $73 \%$; IR $\left(\mathrm{KBr}, \mathrm{cm}^{-1}\right)$ : 3,356 , 3,267 , and $3,256(\mathrm{~m}, \mathrm{~N}-\mathrm{H}$ stretch), 1,674 (s, C=O stretch), 1,589 (s, C=N stretch), 1,497 (s, N-H bend), 1,311 and 1,157 (s, $\mathrm{SO}_{2}$ stretch); ${ }^{1} \mathrm{H}$ NMR $(300 \mathrm{MHz}$, DMSO- $\left.d_{6}\right): \delta 10.23(\mathrm{~s}, 1 \mathrm{H}, \mathrm{C}=\mathrm{CH}), 8.13(\mathrm{~d}, 2 \mathrm{H}, J=7.5$ $\mathrm{Hz}, \mathrm{Ar}-\mathrm{H}), 8.04(\mathrm{~d}, 2 \mathrm{H}, J=8.4 \mathrm{~Hz}, \mathrm{Ar}-\mathrm{H}), 7.95$ (d, 2H, $J=$ 8.4 Hz, Ar-H), 7.77 to 7.80 (m, 2H, Ar-H), 7.60 to $7.62(\mathrm{~m}$, $3 \mathrm{H}$, pyrazole $\left.\mathrm{C}_{5}-\mathrm{H}, \mathrm{Ar}-\mathrm{H}\right), 7.52\left(\mathrm{~s}, \mathrm{ex}, 2 \mathrm{H}, \mathrm{SO}_{2} \mathrm{NH}_{2}\right), 7.46$ (t, $2 \mathrm{H}, J=7.5 \mathrm{~Hz}, \mathrm{Ar}-\mathrm{H}), 7.21$ to $7.23(\mathrm{~m}, 1 \mathrm{H}, \mathrm{Ar}-\mathrm{H}), 2.23$ $\left(\mathrm{s}, 3 \mathrm{H}, \mathrm{CH}_{3}\right)$; DART MS: $\mathrm{m} / z$ 484.30 $[\mathrm{M}+\mathrm{H}]^{+}$, $\mathrm{C}_{26} \mathrm{H}_{21} \mathrm{~N}_{5} \mathrm{O}_{3} \mathrm{SH}^{+}$Calcd. 484.13.

\section{4-\{4-[(3-methyl-5-oxo-1-phenyl-1,5-dihydro-4H-pyrazol-4- ylidene)methyl]-3-(4-methylphenyl)-1H-pyrazol-1-yl\} benzenesulfonamide $(4 b)$}

M.p. $158^{\circ} \mathrm{C}$ to $160^{\circ} \mathrm{C}$, yield $74 \%$; IR $\left(\mathrm{KBr}, \mathrm{cm}^{-1}\right): 3,742$, 3,272, and 3,126 ( $\mathrm{m}, \mathrm{N}-\mathrm{H}$ stretch), 1,682 (s, C=O stretch), 1,589 (s, C=N stretch), 1,497 (s, N-H bend), 1,319 and 1,149 (s, SO $\mathrm{SO}_{2}$ stretch); ${ }^{1} \mathrm{H}$ NMR $(300 \mathrm{MHz}$, DMSO- $\left.d_{6}\right): \delta 10.23(\mathrm{~s}, 1 \mathrm{H}, \mathrm{C}=\mathrm{CH}), 8.14(\mathrm{~d}, 2 \mathrm{H}, J=8.7$ $\mathrm{Hz}, \mathrm{Ar}-\mathrm{H}), 8.04(\mathrm{~d}, 2 \mathrm{H}, J=8.7 \mathrm{~Hz}, \mathrm{Ar}-\mathrm{H}), 7.95(\mathrm{~d}, 2 \mathrm{H}$, $J=7.8 \mathrm{~Hz}$, Ar- $\mathrm{H}), 7.68(\mathrm{~d}, 2 \mathrm{H}, J=7.8 \mathrm{~Hz}$, Ar- $\mathrm{H}), 7.53$ to $7.54\left(\mathrm{~m}, 3 \mathrm{H}\right.$, pyrazole $\left.\mathrm{C}_{5}-\mathrm{H}, \mathrm{SO}_{2} \mathrm{NH}_{2}\right), 7.47(\mathrm{~d}, 2 \mathrm{H}$, $J=7.8 \mathrm{~Hz}, \mathrm{Ar}-\mathrm{H}), 7.43(\mathrm{~d}, 2 \mathrm{H}, J=7.5 \mathrm{~Hz}, \mathrm{Ar}-\mathrm{H})$, 7.21 to $7.24(\mathrm{~m}, 1 \mathrm{H}, \mathrm{Ar}-\mathrm{H}), 2.43\left(\mathrm{~s}, 3 \mathrm{H}, \mathrm{CH}_{3}\right), 2.25$ (s, 3H, $\mathrm{CH}_{3}$ ); DART MS: $m / z 498.30[\mathrm{M}+\mathrm{H}]^{+}$, $\mathrm{C}_{27} \mathrm{H}_{23} \mathrm{~N}_{5} \mathrm{O}_{3} \mathrm{SH}^{+}$Calcd. 498.15. 
4-\{3-(4-methoxyphenyl)-4-[(3-methyl-5-oxo-1-phenyl-1,5dihydro-4H-pyrazol-4-ylidene)methyl]-1H-pyrazol-1-yl\} benzenesulfonamide (4c)

M.p. $156^{\circ} \mathrm{C}$ to $158^{\circ} \mathrm{C}$, yield $76 \%$; IR $\left(\mathrm{KBr}, \mathrm{cm}^{-1}\right): 3,372$, 3,271, and 3,123 (m, N-H stretch), 1,674 (s, C=O stretch), 1,597 (s, C=N stretch), 1,504 (s, N-H bend), 1,319 and 1,157 (s, $\mathrm{SO}_{2}$ stretch); ${ }^{1} \mathrm{H}$ NMR (300 MHz, DMSO- $\left.d_{6}\right): \delta 10.24(\mathrm{~s}, 1 \mathrm{H}, \mathrm{C}=\mathrm{CH}), 8.15(\mathrm{~d}, 2 \mathrm{H}, J=8.1$ $\mathrm{Hz}, \mathrm{Ar}-\mathrm{H}), 8.04$ (d, 2H, $J=7.8 \mathrm{~Hz}, \mathrm{Ar}-\mathrm{H}), 7.97$ (d, $2 \mathrm{H}, J=$ $7.5 \mathrm{~Hz}, \mathrm{Ar}-\mathrm{H}), 7.74$ (d, 2H, J = 7.8 Hz, Ar-H), 7.55 (s, 1H, pyrazole $\left.\mathrm{C}_{5}-\mathrm{H}\right), 7.52$ (s, ex, $\left.2 \mathrm{H}, \mathrm{SO}_{2} \mathrm{NH}_{2}\right), 7.48$ (d, $2 \mathrm{H}, J=$ $8.1 \mathrm{~Hz}, \mathrm{Ar}-\mathrm{H}), 7.16$ to 7.22 (m, 3H, Ar-H), 3.87 (s, 3H, $\left.\mathrm{OCH}_{3}\right), 2.28\left(\mathrm{~s}, 3 \mathrm{H}, \mathrm{CH}_{3}\right)$; DART MS: $m / z 514.29[\mathrm{M}+\mathrm{H}]^{+}$, $\mathrm{C}_{27} \mathrm{H}_{23} \mathrm{~N}_{5} \mathrm{O}_{4} \mathrm{SH}^{+}$Calcd. 514.15.

4-\{4-[(3-methyl-5-oxo-1-phenyl-1,5-dihydro-4H-pyrazol-4ylidene)methyl]-3-(4-fluorophenyl)-1H-pyrazol-1-yl\} benzenesulfonamide (4d)

M.p. $258^{\circ} \mathrm{C}$ to $260^{\circ} \mathrm{C}$, yield $74 \%$; IR $\left(\mathrm{KBr}, \mathrm{cm}^{-1}\right): 3,333$, 3,225 , and 3,132 (m, N-H stretch), 1,674 (s, C=O stretch), 1,597 (s, C=N stretch), 1,504 (s, N-H bend), 1,311 and 1,157 (s, $\mathrm{SO}_{2}$ stretch); ${ }^{1} \mathrm{H}$ NMR (300 MHz, DMSO- $\left.d_{6}\right): \delta 10.25(\mathrm{~s}, 1 \mathrm{H}, \mathrm{C}=\mathrm{CH}), 8.16(\mathrm{~d}, 2 \mathrm{H}, J=8.4$ $\mathrm{Hz}, \mathrm{Ar}-\mathrm{H}), 8.05$ (d, 2H, $J=8.7 \mathrm{~Hz}, \mathrm{Ar}-\mathrm{H}), 7.96$ (d, 2H, $J=$ 8.4 Hz, Ar-H), 7.84 to 7.89 (m, 2H, Ar-H), 7.53 (br s, $3 \mathrm{H}$, pyrazole $\mathrm{C}_{5}-\mathrm{H}, \mathrm{SO}_{2} \mathrm{NH}_{2}$ ), 7.44 to $7.46(\mathrm{~m}, 4 \mathrm{H}, \mathrm{Ar}-\mathrm{H}), 7.20$ to $7.24(\mathrm{~m}, 1 \mathrm{H}, \mathrm{Ar}-\mathrm{H}), 2.28\left(\mathrm{~s}, 3 \mathrm{H}, \mathrm{CH}_{3}\right)$; DART MS: $\mathrm{m} / z$ $502.30[\mathrm{M}+\mathrm{H}]^{+}, \mathrm{C}_{26} \mathrm{H}_{20} \mathrm{FN}_{5} \mathrm{O}_{3} \mathrm{SH}^{+}$Calcd. 502.13.

4-\{3-(4-chlorophenyl)-4-[(3-methyl-5-oxo-1-phenyl-1,5dihydro-4H-pyrazol-4-ylidene)methyl]-1H-pyrazol-1-yl\} benzenesulfonamide (4e)

M.p. $236^{\circ} \mathrm{C}$ to $238^{\circ} \mathrm{C}$, yield $74 \%$; IR $\left(\mathrm{KBr}, \mathrm{cm}^{-1}\right): 3,372$, 3,271 , and 3,123 (m, N-H stretch), 1,674 (s, C=O stretch), 1,589 (s, C=N stretch), 1,497 (s, N-H bend), 1,319 and 1,157 (s, $\mathrm{SO}_{2}$ stretch); ${ }^{1} \mathrm{H}$ NMR (300 MHz, DMSO- $\left.d_{6}\right): \delta 10.23(\mathrm{~s}, 1 \mathrm{H}, \mathrm{C}=\mathrm{CH}), 8.14(\mathrm{~d}, 2 \mathrm{H}, J=8.7$ $\mathrm{Hz}, \mathrm{Ar}-\mathrm{H}), 8.05$ (d, 2H, $J=8.7 \mathrm{~Hz}, \mathrm{Ar}-\mathrm{H}), 7.95$ (d, 2H, $J=$ $8.1 \mathrm{~Hz}, \mathrm{Ar}-\mathrm{H}), 7.83$ (d, 2H, $J=8.4 \mathrm{~Hz}, \mathrm{Ar}-\mathrm{H}), 7.68$ (d, 2H, $J=8.4 \mathrm{~Hz}, \mathrm{Ar}-\mathrm{H}), 7.53\left(\mathrm{~s}, \mathrm{ex}, 2 \mathrm{H}, \mathrm{SO}_{2} \mathrm{NH}_{2}\right), 7.51(\mathrm{~s}, 1 \mathrm{H}$, pyrazole $\left.\mathrm{C}_{5}-\mathrm{H}\right), 7.43(\mathrm{~d}, 2 \mathrm{H}, J=7.8 \mathrm{~Hz}$ Ar-H), 7.21 to 7.24 (m, 1H, Ar-H), 2.27 (s, 3H, $\mathrm{CH}_{3}$ ); DART MS: $m / z$ 518.26/ $520.25[\mathrm{M}+\mathrm{H}]^{+} /[\mathrm{M}+\mathrm{H}+2]^{+}, \mathrm{C}_{26} \mathrm{H}_{20} \mathrm{ClN}_{5} \mathrm{O}_{3} \mathrm{SH}^{+}$Calcd. $518.10 / 520.10$.

4-\{3-(4-bromophenyl)-4-[(3-methyl-5-oxo-1-phenyl-1,5dihydro-4H-pyrazol-4-ylidene)methyl]-1H-pyrazol-1-yl\} benzenesulfonamide (4f)

M.p. $162^{\circ} \mathrm{C}$ to $165^{\circ} \mathrm{C}$, yield $70 \%$; IR $\left(\mathrm{KBr}, \mathrm{cm}^{-1}\right): 3,373$, 3,261, and 3,123 ( $\mathrm{m}, \mathrm{N}-\mathrm{H}$ stretch), 1,682 (s, C=O stretch), 1,597 (s, C=N stretch), 1,497 (s, N-H bend), 1,311 and 1,157 (s, $\mathrm{SO}_{2}$ stretch); ${ }^{1} \mathrm{H}$ NMR (300 MHz, DMSO- $\left.d_{6}\right): \delta 10.20(\mathrm{~s}, 1 \mathrm{H}, \mathrm{C}=\mathrm{CH}), 8.11(\mathrm{~d}, 2 \mathrm{H}, J=8.7$ $\mathrm{Hz}, \mathrm{Ar}-\mathrm{H}), 8.04(\mathrm{~d}, 2 \mathrm{H}, J=8.7 \mathrm{~Hz}, \mathrm{Ar}-\mathrm{H}), 7.92$ to 7.99 (m, 3H, Ar-H), 7.80 (d, 2H, $J=8.1 \mathrm{~Hz}, \mathrm{Ar}-\mathrm{H}), 7.67$ to 7.74 (m, 3H, Ar-H), $7.61\left(\mathrm{~s}, 1 \mathrm{H}\right.$, pyrazole $\left.\mathrm{C}_{5}-\mathrm{H}\right), 7.54$ (s, ex, $2 \mathrm{H}$, $\left.\mathrm{SO}_{2} \mathrm{NH}_{2}\right), 7.20$ to $7.26(\mathrm{~m}, 1 \mathrm{H}, \mathrm{Ar}-\mathrm{H}), 2.24\left(\mathrm{~s}, 3 \mathrm{H}, \mathrm{CH}_{3}\right)$; DART MS: $m / z 562.20 / 564.19[\mathrm{M}+\mathrm{H}]^{+} /[\mathrm{M}+\mathrm{H}+2]^{+}$, $\mathrm{C}_{26} \mathrm{H}_{20} \mathrm{BrN}_{5} \mathrm{O}_{3} \mathrm{SH}^{+}$Calcd. 562.05/564.05.

4-\{4-[(3-methyl-5-oxo-1-phenyl-1,5-dihydro-4H-pyrazol-4ylidene)methyl]-3-(4-nitrophenyl)-1H-pyrazol-1-yl\} benzenesulfonamide $(4 \mathrm{~g})$

M.p. $310^{\circ} \mathrm{C}$ to $312^{\circ} \mathrm{C}$, yield $73 \%$; IR $\left(\mathrm{KBr}, \mathrm{cm}^{-1}\right): 3,371$, 3,271 , and 3,124 (m, N-H stretch), 1,674 (s, C=O stretch), 1,589 (s, C=N stretch), 1,497 (s, N-H bend), 1,335 and 1,157 (s, $\mathrm{SO}_{2}$ stretch); ${ }^{1} \mathrm{H}$ NMR (300 MHz, DMSO- $\left.d_{6}\right): \delta$ $10.23(\mathrm{~s}, 1 \mathrm{H}, \mathrm{C}=\mathrm{CH}), 8.44(\mathrm{~d}, 2 \mathrm{H}, J=8.4 \mathrm{~Hz}, \mathrm{Ar}-\mathrm{H}), 8.16$ $(\mathrm{d}, 2 \mathrm{H}, J=8.4 \mathrm{~Hz}, \mathrm{Ar}-\mathrm{H}), 8.04$ to $8.11(\mathrm{~m}, 4 \mathrm{H}, \mathrm{Ar}-\mathrm{H}), 7.94$ (d, $2 \mathrm{H}, J=7.8 \mathrm{~Hz}, \mathrm{Ar}-\mathrm{H}), 7.56\left(\mathrm{~s}, 1 \mathrm{H}\right.$, pyrazole $\left.\mathrm{C}_{5}-\mathrm{H}\right), 7.54$ (s, ex, $2 \mathrm{H}, \mathrm{SO}_{2} \mathrm{NH}_{2}$ ), 7.43 to $7.48(\mathrm{~m}, 2 \mathrm{H}, \mathrm{Ar}-\mathrm{H}), 7.21$ to $7.30(\mathrm{~m}, 1 \mathrm{H}, \mathrm{Ar}-\mathrm{H}), 2.29\left(\mathrm{~s}, 3 \mathrm{H}, \mathrm{CH}_{3}\right)$; DART MS: $\mathrm{m} / z$ 529.28 $[\mathrm{M}+\mathrm{H}]^{+}, \mathrm{C}_{26} \mathrm{H}_{20} \mathrm{~N}_{6} \mathrm{O}_{5} \mathrm{SH}^{+}$Calcd. 529.12.

4-[4-(\{1-[4-(aminosulfonyl)phenyl]-3-methyl-5-oxo-1,5dihydro-4H-pyrazol-4-ylidene\}methyl)-3-phenyl-1H-pyrazol1-yl]benzenesulfonamide (5a)

M.p. $248^{\circ} \mathrm{C}$ to $252^{\circ} \mathrm{C}$, yield $73 \%$; IR $\left(\mathrm{KBr}, \mathrm{cm}^{-1}\right): 3,340$, 3,271 , and 3,217 (m, N-H stretch), 1,674 (s, C=O stretch), 1,589 (s, C=N stretch), 1,504 (s, N-H bend), 1,335 and 1165 (s, $\mathrm{SO}_{2}$ stretch); ${ }^{1} \mathrm{H}$ NMR (300 MHz, DMSO- $\left.d_{6}\right): \delta 10.21(\mathrm{~s}, 1 \mathrm{H}, \mathrm{C}=\mathrm{CH}), 8.14$ to $8.19(\mathrm{~m}, 4 \mathrm{H}$, Ar-H), 8.05 (d, 2H, $J=8.4 \mathrm{~Hz}, \mathrm{Ar}-\mathrm{H}), 7.91$ (d, 2H, $J=8.7$ $\mathrm{Hz}, \mathrm{Ar}-\mathrm{H}), 7.79$ to 7.80 (m, 2H, Ar-H), 7.59 to 7.63 (m, $4 \mathrm{H}$, pyrazole $\left.\mathrm{C}_{5}-\mathrm{H}, \mathrm{Ar}-\mathrm{H}\right), 7.54$ (s, ex, $2 \mathrm{H}, \mathrm{SO}_{2} \mathrm{NH}_{2}$ ), 7.36 (s, ex, $2 \mathrm{H}, \mathrm{SO}_{2} \mathrm{NH}_{2}$ ), 2.28 (s, $3 \mathrm{H}, \mathrm{CH}_{3}$ ); DART MS: $\mathrm{m} / z$ 563.28 $[\mathrm{M}+\mathrm{H}]^{+}, \mathrm{C}_{26} \mathrm{H}_{22} \mathrm{~N}_{6} \mathrm{O}_{5} \mathrm{~S}_{2} \mathrm{H}^{+}$Calcd. 563.10.

[4-(\{1-[4-(aminosulfonyl)phenyl]-3-methyl-5-oxo-1,5-dihydro4H-pyrazol-4-ylidene\}methyl)-3-(4-methylphenyl)-1Hpyrazol-1-yl]benzenesulfonamide (5b)

M.p. $282^{\circ} \mathrm{C}$ to $285^{\circ} \mathrm{C}$, yield $75 \%$; IR $\left(\mathrm{KBr}, \mathrm{cm}^{-1}\right): 3,340$, 3,248 (m, N-H stretch), 1,682 (s, C=O stretch), 1,589 (s, $\mathrm{C}=\mathrm{N}$ stretch), 1,497 (s, N-H bend), 1,304 and 1,149 (s, $\mathrm{SO}_{2}$ stretch); ${ }^{1} \mathrm{H}$ NMR (300 MHz, DMSO-d $): \delta 10.19$ (s, $1 \mathrm{H}, \mathrm{C}=\mathrm{CH}), 8.13$ to $8.19(\mathrm{~m}, 4 \mathrm{H}, \mathrm{Ar}-\mathrm{H}), 8.05(\mathrm{~d}$, $2 \mathrm{H}, J=8.4 \mathrm{~Hz}, \mathrm{Ar}-\mathrm{H}), 7.92(\mathrm{~d}, 2 \mathrm{H}, J=8.4 \mathrm{~Hz}, \mathrm{Ar}-\mathrm{H})$, $7.68(\mathrm{~d}, 2 \mathrm{H}, J=7.8 \mathrm{~Hz}, \mathrm{Ar}-\mathrm{H}), 7.58(\mathrm{~s}, 1 \mathrm{H}$, pyrazole $\left.\mathrm{C}_{5}-\mathrm{H}\right), 7.52\left(\mathrm{~s}, \mathrm{ex}, 2 \mathrm{H}, \mathrm{SO}_{2} \mathrm{NH}_{2}\right), 7.42(\mathrm{~d}, 2 \mathrm{H}, J=7.5$ $\mathrm{Hz}, \mathrm{Ar}-\mathrm{H}$ ), 7.34 (s, ex, $\left.2 \mathrm{H}, \mathrm{SO}_{2} \mathrm{NH}_{2}\right), 2.43\left(\mathrm{~s}, 3 \mathrm{H}, \mathrm{CH}_{3}\right)$, $2.28\left(\mathrm{~s}, 3 \mathrm{H}, \mathrm{CH}_{3}\right)$; DART MS: $m / z 577.32[\mathrm{M}+\mathrm{H}]^{+}$, $\mathrm{C}_{27} \mathrm{H}_{24} \mathrm{~N}_{6} \mathrm{O}_{5} \mathrm{~S}_{2} \mathrm{H}^{+}$Calcd. 577.12.

4-[4-(\{1-[4-(aminosulfonyl)phenyl]-3-methyl-5-oxo-1,5dihydro-4H-pyrazol-4-ylidene\}methyl)-3-(4-methoxyphenyl)1H-pyrazol-1-yl]benzenesulfonamide (5c)

M.p. $320^{\circ} \mathrm{C}$ to $326^{\circ} \mathrm{C}$, yield $74 \%$; IR $\left(\mathrm{KBr}, \mathrm{cm}^{-1}\right): 3,317$, 3,232, 3,148 and 3,086 (m, N-H stretch), 1,674 (s, C=O 
stretch), 1,589 (s, C=N stretch), 1,504 (s, N-H bend), 1,335 and 1157 (s, $\mathrm{SO}_{2}$ stretch); ${ }^{1} \mathrm{H}$ NMR (300 MHz, DMSO- $\left.d_{6}\right): \delta 10.18(\mathrm{~s}, 1 \mathrm{H}, \mathrm{C}=\mathrm{CH}), 8.12$ to $8.19(\mathrm{~m}, 4 \mathrm{H}$, Ar-H), 8.04 (d, 2H, $J=8.7 \mathrm{~Hz}, \mathrm{Ar}-\mathrm{H}), 7.91$ (d, 2H, $J=8.7$ $\mathrm{Hz}, \mathrm{Ar}-\mathrm{H}), 7.73$ (d, 2H, $J=8.7 \mathrm{~Hz}, \mathrm{Ar}-\mathrm{H}), 7.57$ (s, 1H, pyrazole $\mathrm{C}_{5}-\mathrm{H}$ ), 7.53 (s, ex, $2 \mathrm{H}, \mathrm{SO}_{2} \mathrm{NH}_{2}$ ), 7.36 (s, ex, $2 \mathrm{H}$, $\left.\mathrm{SO}_{2} \mathrm{NH}_{2}\right), 7.16(\mathrm{~d}, 2 \mathrm{H}, J=8.4 \mathrm{~Hz}, \mathrm{Ar}-\mathrm{H}), 3.86(\mathrm{~s}, 3 \mathrm{H}$, $\left.\mathrm{OCH}_{3}\right), 2.28\left(\mathrm{~s}, 3 \mathrm{H}, \mathrm{CH}_{3}\right)$; DART MS: $m / z 593.30[\mathrm{M}+\mathrm{H}]^{+}$, $\mathrm{C}_{27} \mathrm{H}_{24} \mathrm{~N}_{6} \mathrm{O}_{6} \mathrm{~S}_{2} \mathrm{H}^{+}$Calcd. 593.12.

\section{4-[4-(\{1-[4-(aminosulfonyl)phenyl]-3-methyl-5-oxo-1,5-} dihydro-4H-pyrazol-4-ylidene\}methyl)-3-(4-fluorophenyl)- $1 \mathrm{H}$ pyrazol-1-yl]benzenesulfonamide (5d)

M.p. $208^{\circ} \mathrm{C}$ to $210^{\circ} \mathrm{C}$, yield $71 \%$; IR $\left(\mathrm{KBr}, \mathrm{cm}^{-1}\right): 3,317$, 3,202, 3,148 and 3,070 (m, N-H stretch), 1,674 (s, C=O stretch), 1,589 (s, C=N stretch), 1,504 (s, N-H bend), 1,335 and 1,157 (s, $\mathrm{SO}_{2}$ stretch); ${ }^{1} \mathrm{H}$ NMR (300 MHz, DMSO- $\left.d_{6}\right): \delta 10.20(\mathrm{~s}, 1 \mathrm{H}, \mathrm{C}=\mathrm{CH}), 8.14$ to $8.19(\mathrm{~m}, 4 \mathrm{H}$, Ar-H), 8.05 (d, 2H, J = 8.7 Hz, Ar-H), 7.91 (d, 2H, $J=$ $8.7 \mathrm{~Hz}, \mathrm{Ar}-\mathrm{H}), 7.86$ (dd, 2H, $J=8.1,5.4 \mathrm{~Hz}$ Ar-H), 7.55 (br s, $3 \mathrm{H}$, pyrazole $\mathrm{C}_{5}-\mathrm{H}, \mathrm{SO}_{2} \mathrm{NH}_{2}$ ), 7.43 to 7.49 (m, $2 \mathrm{H}$, Ar-H), 7.37 (s, ex, 2H, $\left.\mathrm{SO}_{2} \mathrm{NH}_{2}\right), 2.29$ (s, 3H, $\left.\mathrm{CH}_{3}\right)$; DART MS: $m / z 581.26[\mathrm{M}+\mathrm{H}]^{+}, \mathrm{C}_{26} \mathrm{H}_{21} \mathrm{FN}_{6} \mathrm{O}_{5} \mathrm{~S}_{2} \mathrm{H}^{+}$ Calcd. 581.10

\section{4-[4-(\{1-[4-(aminosulfonyl)phenyl]-3-methyl-5-oxo-1,5- dihydro-4H-pyrazol-4-ylidene\}methyl)-3-(4-chlorophenyl)- 1H-pyrazol-1-yl]benzenesulfonamide (5e)}

M.p. $318^{\circ} \mathrm{C}$ to $320^{\circ} \mathrm{C}$, yield $72 \%$; IR $\left(\mathrm{KBr}, \mathrm{cm}^{-1}\right): 3,340$, 3,256, 3,148 and 3,086 (m, N-H stretch), 1,682 (s, C=O stretch), 1,597 (s, C=N stretch), 1,497 (s, N-H bend), 1,335 and 1,157 (s, $\mathrm{SO}_{2}$ stretch); ${ }^{1} \mathrm{H}$ NMR (300 MHz, DMSO- $\left.d_{6}\right): \delta 10.20$ (s, $\left.1 \mathrm{H}, \mathrm{C}=\mathrm{CH}\right), 8.15$ to $8.19(\mathrm{~m}, 4 \mathrm{H}$, Ar-H), 8.06 (d, 2H, J = 8.4 Hz, Ar-H), 7.92 (d, 2H, J = $8.7 \mathrm{~Hz}, \mathrm{Ar}-\mathrm{H}), 7.84$ (d, 2H, $J=8.1 \mathrm{~Hz}, \mathrm{Ar}-\mathrm{H}), 7.68$ (d, $2 \mathrm{H}, J=8.1 \mathrm{~Hz}, \mathrm{Ar}-\mathrm{H}), 7.58\left(\mathrm{~s}, 1 \mathrm{H}\right.$, pyrazole $\left.\mathrm{C}_{5}-\mathrm{H}\right), 7.54$ (s, ex, $2 \mathrm{H}, \mathrm{SO}_{2} \mathrm{NH}_{2}$ ), 7.36 (s, ex, $2 \mathrm{H}, \mathrm{SO}_{2} \mathrm{NH}_{2}$ ), 2.31 (s, $3 \mathrm{H}, \mathrm{CH}_{3}$ ); DART MS: $m / z$ 597.25/599.25 $[\mathrm{M}+\mathrm{H}]^{+} /[\mathrm{M}+$ $\mathrm{H}+2]^{+}, \mathrm{C}_{26} \mathrm{H}_{21} \mathrm{ClN}_{6} \mathrm{O}_{5} \mathrm{~S}_{2} \mathrm{H}^{+}$Calcd. 597.07/599.07.

\section{4-[4-(\{1-[4-(aminosulfonyl)phenyl]-3-methyl-5-oxo-1,5- dihydro-4H-pyrazol-4-ylidene\}methyl)-3-(4-bromophenyl)- 1H-pyrazol-1-yl]benzenesulfonamide (5f)}

M.p. $330^{\circ} \mathrm{C}$ to $332^{\circ} \mathrm{C}$, yield $69 \%$; IR $\left(\mathrm{KBr}, \mathrm{cm}^{-1}\right): 3,742$, 3,333 and 3,256 (m, N-H stretch), 1,682 (s, C=O stretch), 1,597 (s, C=N stretch), 1,497 (s, N-H bend), 1,335 and 1,157 (s, $\mathrm{SO}_{2}$ stretch); ${ }^{1} \mathrm{H}$ NMR (300 MHz, DMSO- $\left.d_{6}\right): \delta 10.19$ (s, $\left.1 \mathrm{H}, \mathrm{C}=\mathrm{CH}\right), 8.15$ to $8.19(\mathrm{~m}, 4 \mathrm{H}$, Ar-H), 8.06 (d, 2H, J = 8.4 Hz, Ar-H), 7.92 (d, 2H, J = 8.4 Hz, Ar-H), 7.82 (d, 2H, J = $7.8 \mathrm{~Hz}, \mathrm{Ar}-\mathrm{H}), 7.76$ (d, $2 \mathrm{H}, J=7.8 \mathrm{~Hz}, \mathrm{Ar}-\mathrm{H}), 7.57$ (s, $1 \mathrm{H}$, pyrazole $\left.\mathrm{C}_{5}-\mathrm{H}\right), 7.54$ (s, ex, $2 \mathrm{H}, \mathrm{SO}_{2} \mathrm{NH}_{2}$ ), 7.35 (s, ex, $2 \mathrm{H}, \mathrm{SO}_{2} \mathrm{NH}_{2}$ ), 2.31 (s, $3 \mathrm{H}, \mathrm{CH}_{3}$ ); DART MS: $m / z$ 641.19/643.20 $[\mathrm{M}+\mathrm{H}]^{+} /[\mathrm{M}+$ $\mathrm{H}+2]^{+}, \mathrm{C}_{26} \mathrm{H}_{21} \mathrm{BrN}_{6} \mathrm{O}_{5} \mathrm{~S}_{2} \mathrm{H}^{+}$Calcd. 641.02/643.02. 4-[4-(\{1-[4-(aminosulfonyl)phenyl]-3-methyl-5-oxo-1,5dihydro-4H-pyrazol-4-ylidene\}methyl)-3-(4-nitrophenyl)-1Hpyrazol-1-yl]benzenesulfonamide (5g)

M.p. $298^{\circ} \mathrm{C}$ to $300^{\circ} \mathrm{C}$, yield $71 \%$; IR $\left(\mathrm{KBr}, \mathrm{cm}^{-1}\right)$ : 3,348 , 3,248, 3,132 and 3,109 (m, N-H stretch), 1,682 (s, C=O stretch), 1,589 (s, C=N stretch), 1,528 (s, N-H bend), 1,335 and 1,157 (s, $\mathrm{SO}_{2}$ stretch); ${ }^{1} \mathrm{H}$ NMR (300 MHz, DMSO- $\left.d_{6}\right): \delta 10.21(\mathrm{~s}, 1 \mathrm{H}, \mathrm{C}=\mathrm{CH}), 8.45(\mathrm{~d}, 2 \mathrm{H}, J=8.4$ $\mathrm{Hz}, \mathrm{Ar}-\mathrm{H}), 8.16$ to 8.19 (m, 4H, Ar-H), 8.11 (d, 2H, J = $9.0 \mathrm{~Hz}, \mathrm{Ar}-\mathrm{H}), 8.07$ (d, 2H, $J=8.7 \mathrm{~Hz}, \mathrm{Ar}-\mathrm{H}), 7.92$ (d, $2 \mathrm{H}, J=8.7 \mathrm{~Hz}, \mathrm{Ar}-\mathrm{H}), 7.62\left(\mathrm{~s}, 1 \mathrm{H}\right.$, pyrazole $\left.\mathrm{C}_{5}-\mathrm{H}\right), 7.55$ (s, ex, $2 \mathrm{H}, \mathrm{SO}_{2} \mathrm{NH}_{2}$ ), 7.36 (s, ex, $2 \mathrm{H}, \mathrm{SO}_{2} \mathrm{NH}_{2}$ ), 2.33 (s, $3 \mathrm{H}, \mathrm{CH}_{3}$ ); DART MS: $m / z 608.27[\mathrm{M}+\mathrm{H}]^{+}, \mathrm{C}_{26} \mathrm{H}_{21}$ $\mathrm{N}_{7} \mathrm{O}_{7} \mathrm{~S}_{2} \mathrm{H}^{+}$Calcd. 608.09.

\section{Conclusions}

In conclusion, we have presented the novel 4-arylidene pyrazole derivatives bearing benzenesulfonamide moiety as potential antimicrobial agents. The reported compounds were conveniently prepared by Knovenagel condensation of 4-formyl pyrazoles with pyrazolones. Some of the tested compounds displayed excellent antibacterial properties against Gram-positive bacteria (B. subtilis and $S$. aureus). For instance, compounds $4 \mathbf{a}$ and $5 \mathbf{a}$ were found to be more effective than the reference drug ciprofloxacin. However, against Gram-negative bacteria ( $P$. fluorescens and E. coli), the level of activity shown by the tested compounds was found to be significantly low as only 2 of the 14 tested compounds displayed activities comparable to the reference drug against $P$. fluorescens, while none of the compounds were found to be highly effective against E. coli. All the tested compounds showed moderate antifungal activity against C. albicans, while two compounds showed activity better than the reference drug against $S$. cerevisiae. In short, the reported compounds showed remarkable potential as antimicrobial agents and warranted further investigation of their mechanism of actions and binding site. The studies regarding these aspects are being planned with the help of a prospective collaborator.

\section{Abbreviations \\ DMSO: Dimethylsulfoxide; MIC: Minimum inhibitory concentration; MTCC: Microbial-type culture collection.}

\section{Competing interests}

The authors declare that they have no competing interests.

\section{Acknowledgements}

Defence Research and Development Organization (DRDO), New Delhi, is thankfully acknowledged for the financial support in the form of a research project. One of the authors (PK) is grateful to the Haryana State Council for Science and Technology (HSCST), Panchkula (Haryana), India, for the award of Junior Research Fellowship. The authors are thankful to the Sophisticated Analytical Instrument Facility, Central Drug Research Institute, Lucknow, for the mass spectra analysis. 


\section{Author details}

${ }^{1}$ Department of Chemistry, Kurukshetra University, Kurukshetra 136119, India. ${ }^{2}$ Department of Microbiology, Kurukshetra University, Kurukshetra 136119 India.

Received: 4 June 2013 Accepted: 20 August 2013 Published: 28 August 2013

\section{References}

1. Bondock S, Fadaly W, Metwally MA (2010) Synthesis and antimicrobial activity of some new thiazole, thiophene and pyrazole derivatives containing benzothiazole moiety. Eur J Med Chem 45:3692-3702

2. Isloor AM, Kalluraya B, Shetty P (2009) Regioselective reaction: synthesis, characterization, and pharmacological studies of some new mannich bases derived from 1,2,4-triazoles. Eur J Med Chem 44:3784-3787

3. Prakash O, Hussain K, Kumar R, Wadhwa D, Sharma C, Aneja KR (2011) Synthesis and antimicrobial evaluation of new 1,4-dihydro-4pyrazolylpyridines and 4-pyrazolylpyridines. Org Med Chem Lett 1:1-5

4. Aneja DK, Lohan P, Arora S, Sharma C, Aneja KR, Prakash O (2011) Synthesis of new pyrazolyl-2, 4-thiazolidinediones as antibacterial and antifungal agents. Org Med Chem Lett 1:1-15

5. El-Sayed MAA, Abdel-Aziz NI, Abdel-Aziz AAM, El-Azab AS, ElTahir KEH (2012) Synthesis, biological evaluation and molecular modeling study of pyrazole and pyrazoline derivatives as selective COX-2 inhibitors and antiinflammatory agents. Bioorg Med Chem 20:3306-3316

6. Singh SK, Saibaba V, Rao KS, Reddy PG, Daga PR, Rajjak SA, Misra P, Rao YK (2005) Synthesis and SAR/3D-QSAR studies on the COX-2 inhibitory activity of 1,5-diarylpyrazoles to validate the modified pharmacophore. Eur J Med Chem 40:977-990

7. Lee KY, Kim JM, Kim JN (2003) Regioselective synthesis of 1,3,4,5tetrasubstituted pyrazoles from Baylis-Hillman adducts. Tetrahedron Lett 44:6737-6740

8. Abdel-Aziz M, Abuo-Rahma El-Din AG, Hassan AA (2009) Synthesis of novel pyrazole derivatives and evaluation of their antidepressant and anticonvulsant activities. Eur J Med Chem 44:3480-3487

9. Hashem Al, Youssef ASA, Kandeel KA, Abou-Elmagd WSI (2007) Conversion of some 2(3H)-furanones bearing a pyrazolyl group into other heterocyclic systems with a study of their antiviral activity. Eur J Med Chem 42:934-939

10. Lv P-C, Li H-Q, Sun J, Zhou Y, Zhu H-L (2010) Synthesis and biological evaluation of pyrazole derivatives containing thiourea skeleton as anticancer agents. Bioorg Med Chem 18:4606-4614

11. Sridhar R, Perumal PT, Etti S, Shanmugan PMN, Prabavathy VR, Mathivanan N (2004) Design, synthesis and anti-microbial activity of $1 \mathrm{H}$-pyrazole carboxylates. Bioorg Med Chem Lett 14:6035-6040

12. Sharma PK, Kumar S, Kumar P, Kaushik P, Kaushik D, Dhingra Y, Aneja KR (2010) Synthesis and biological evaluation of some pyrazolylpyrazolines as anti-inflammatory-antimicrobial agents. Eur J Med Chem 45:2650-2655

13. Rathelot $P$, Azas N, El-Kashef H, Delmas F, Giorgio CD, Timon-David P Maldonado J, Vanelle P (2002) 1,3-Diphenylpyrazoles: synthesis and antiparasitic activities of azomethine derivatives. Eur J Med Chem 37:671-679

14. Li X, Liu JL, Yang XH, Lu X, Zhao TT, Gong HB, HI Z (2012) Synthesis, biological evaluation and molecular docking studies of 3-(1,3-diphenyl-1Hpyrazol-4yl)-N-phenylacrylamide derivatives as inhibitors of HDAC activity. Bioorg Med Chem 20(14):4430-4436

15. Uramaru N, Shigematsu H, Toda A, Eyanagi R, Kitamura S, Ohta S (2010) Design, synthesis and pharmacological activity of non allergenic pyrazolone type antipyretic analgesics. J Med Chem 53:8727-8733

16. Thaker KM, Ghetiya RM, Tala SD, Dodiya BL, Joshi KA, Dubal KL, Joshi HS (2011) Synthesis of oxadiazoles and pyrazolones as a antimycobacterial and antimicrobial agents. Indian J Chem 50B:738-744

17. Chande MS, Barve PA, Suryanarayan V (2007) Synthesis and antimicrobial activity of novel spiro compounds with pyrazolone and pyrazolthione moiety. J Hetero Chem 44:49-53

18. Mariappan G, Saha BP, Satharson L, Haldar A (2010) Synthesis and bioactivity evaluation of pyrazolone derivatives. Indian J Chem 49B:1671-1674

19. Wang XH, Wang XK, Liang YJ, Shi Z, Zhang JY, Chen LM, Fu LW (2010) A cell-based screen for anticancer activity of 13 pyrazolone derivatives. Chin Cancer 29(12):980-987
20. Chen T, Benmohamed R, Kim J, Smith K, Amanta D, Morimoto Rl, Kirsch DR, Ferrante RJ, Silverman RB (2012) ADME-guided design and synthesis of aryloxanyl pyrazolone derivatives to block mutant superoxide dismutase 1 (SOD1) cytotoxicity and protein aggregation: potential application for the treatment of amyotrophic lateral sclerosis. J Med Chem 55(1):515-527

21. Sharma PK, Chandak N, Kumar P, Sharma C, Aneja KR (2011) Synthesis and biological evaluation of some 4-functionalized pyrazoles as antimicrobial agents. Eur J Med Chem 46:1425-1432

22. Sharma PK, Singh K, Kumar S, Kumar P, Dhawan SN, Lal S, Ulbrich H, Dannhardt G (2011) Synthesis and anti-inflammatory evaluation of some pyrazolo[3,4-b] pyridines. Med Chem Res 20:239-244

23. Kumar P, Chandak N, Kaushik P, Sharma C, Kaushik D, Aneja KR, Sharma PK (2012) Synthesis and biological evaluation of some pyrazole derivatives as anti-inflammatory-antibacterial agents. Med Chem Res 21:3396-3405

24. Sharma PK, Kumar S, Kumar P, Kaushik P, Sharma C, Kaushik D, Aneja KR (2012) Synthesis of 1-(4-aminosulfonylphenyl)-3,5-diarylpyrazoline derivatives as potent anti-inflammatory and antimicrobial agent. Med Chem Res 21:2945-2954

25. Kumar S, Namkung W, Verkman AS, Sharma PK (2012) Novel 5-substituted benzyloxy-2-arylbenzofuran-3-carboxylic acid calcium activated chloride channel inhibitors. Bioorg Med Chem 20:4237-4244

26. Chandak N, Bhardwaj JK, Sharma RK, Sharma PK (2012) Inhibitors of apoptosis in testicular germ cells: synthesis and biological evaluation of some novel IBTs bearing sulfonamide moiety. Eur J Med Chem 59:203-208

27. Chandak N, Kumar P, Sharma C, Aneja KR, Sharma PK (2012) Synthesis and biological evaluation of some novel thiazolylhydrazinomethylidene ferrocenes as antimicrobial agents. Lett Drug Des Discov 9(1):63-68

28. Perez C, Pauli M, Bazevque P (1990) An antibiotic assay by the agar well diffusion method. Acta Biol Med Exp 15:113-115

29. Andrews JM (2001) Determination of minimum inhibitory concentrations. J Antimicrob Chemother 48:5-16

30. Knorr $L$ (1884) Einwirkung von acetessigester auf hydrazinchinizinderivate. Chem Ber 17:546-552

31. Knorr $L$ (1886) Synthetische versuche mit dem acetessigester II mittheilung: ueberführung des diacetbernsteinsäureesters und des acetessigesters in pyrrolderivate. Ann Chem 236:290-336

doi:10.1186/2191-2858-3-9

Cite this article as: Khloya et al:: Synthesis of some novel 4-arylidene pyrazoles as potential antimicrobial agents. Organic and Medicinal Chemistry Letters 2013 3:9.

\section{Submit your manuscript to a SpringerOpen ${ }^{\odot}$ journal and benefit from:}

- Convenient online submission

- Rigorous peer review

- Immediate publication on acceptance

- Open access: articles freely available online

- High visibility within the field

- Retaining the copyright to your article

Submit your next manuscript at springeropen.com 